\section{The guard and the gate}

The overwhelming quantity of information that is gathered by our sensory organs creates a challenge for the brain, as it must ensure that we attend to the information that is most relevant for the task in hand.

The responses of cortical neurons to stimuli to which attention is directed are thus enhanced; however, the mechanisms by which this modulation arises are poorly understood. McAlonan et al. shed light on the early stages of visual processing that are modified by attention.

Sensory information is relayed to the cortex by the thalamus, where it is thought that initial processing events take place. The authors focused on two thalamic regions: the lateral geniculate nucleus (LGN) - known as the 'gateway to the cortex' which receives projections from retinal neurons and in turn projects to the cortex, and the thalamic reticular nucleus (TRN), which Crick termed the 'guardian of the gateway' owing to its inhibitory input to the LGN. They recorded the activity of individual neurons in these regions in monkeys during an attentional task. In each trial, the monkey's attention was directed into or out of the neuron's receptive field, after which a stimulus appeared within the receptive field.

For LGN neurons, directing attention into the receptive field resulted in an increase in the initial response to the stimulus, whereas for the TRN, directing attention into the receptive field decreased the neurons' initial response to the stimulus. Further analysis showed that the modulation of the activity of the TRN neurons by attention occurred slightly earlier than the modulation of neuronal activity in the LGN, which is consistent with a model in which inhibitory inputs from the TRN modulate LGN activity. A later period of modulated activity was also seen in the LGN but not the TRN, suggesting the existence of an additional route through which attention modulates LGN activity. The authors speculate that this might reflect feedback from the cortex.

These findings suggest that incoming visual information is initially modified at the 'gateway' by the activity of the TRN, as proposed by Crick. Further work will be required to determine the extent to which subsequent cortical activity is modified by these events.

ORIGINAL RESEARCH PAPER McAlonan, K Cavanaugh, J. \& Wurtz, R. H. Guarding the gateway to cortex with attention in visual thalamus. Nature 5 Oct 2008

(doi:10.1038/nature07382)

FURTHER READING Crick, F. Function of the reticular complex: the searchlight hypothesis. Proc. Natl Acad. Sci. USA 81, 4586-4590 (1984)

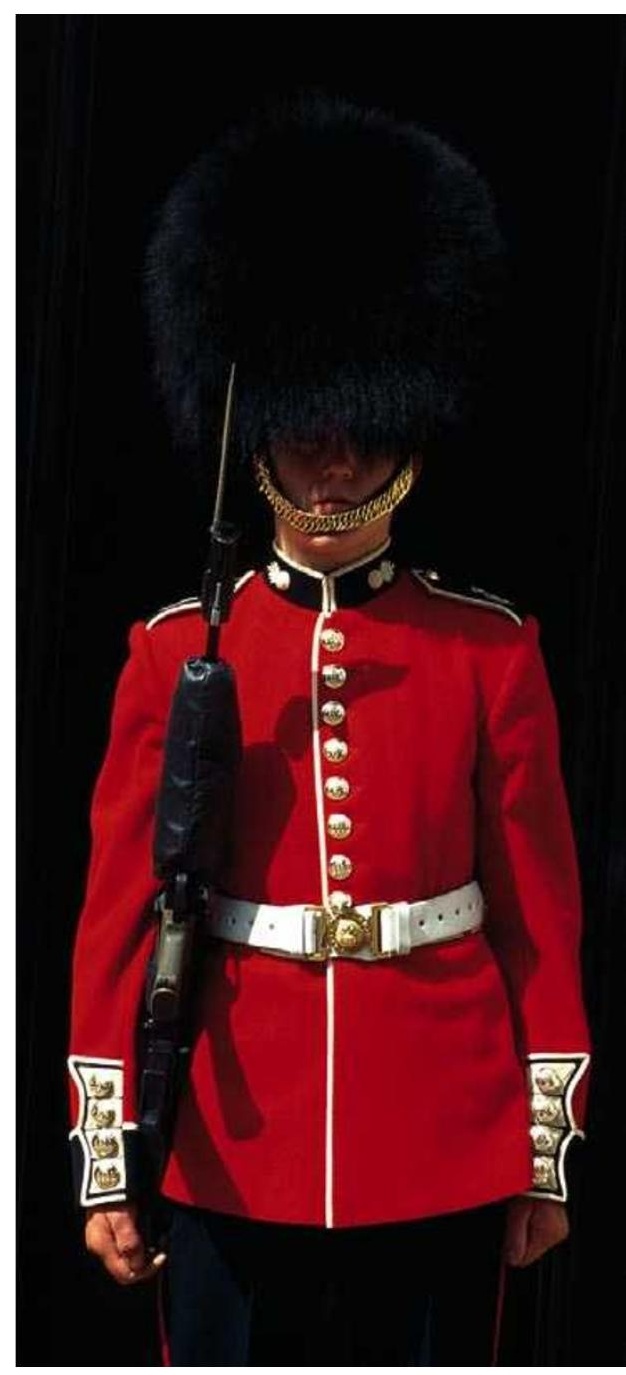

